\title{
Identification of a novel distal enhancer in human adiponectin gene
}

\author{
Katsumori Segawa ${ }^{1,2,3}$, Morihiro Matsuda ${ }^{1,4,5}$, Atsunori Fukuhara ${ }^{1}$, Kentaro Morita ${ }^{1}$, Yosuke Okuno ${ }^{1}$, \\ Ryutaro Komuro ${ }^{1}$ and lichiro Shimomura ${ }^{1}$ \\ ${ }^{1}$ Department of Metabolic Medicine, Graduate School of Medicine, Osaka University, 2-2 Yamadaoka, Suita-shi, Osaka 565-0871, Japan \\ ${ }^{2}$ Graduate School of Frontier Bioscience, Osaka University, Osaka, 565-0871, Japan \\ ${ }^{3} J a p a n$ Society for the Promotion of Science Research, Tokyo 102-8742, Japan \\ ${ }^{4}$ Institute of Clinical Research, National Hospital Organization Kure Medical Center, 3-1 Aoyama-cho, Kure-shi, Hiroshima 737-0023, Japan \\ ${ }^{5}$ Department of Internal Medicine, National Hospital Organization Kure Medical Center, Kure 737-0023, Japan \\ (Correspondence should be addressed to M Matsuda; Email: mmatsuda@imed2.med.osaka-u.ac.jp)
}

\begin{abstract}
Adiponectin is exclusively expressed in adipose tissue and secreted from adipocytes, and shows anti-diabetic and antiatherogenic properties. However, the precise transcriptional mechanism of adiponectin remains elusive. In this study, the $5^{\prime}$ flanking promoter region of human adiponectin gene was analyzed using UCSC genome browser, and a $10390-\mathrm{bp}$ fragment, containing an evolutionally conserved region among species, was investigated. The luciferase reporter assay using this fragment identified a novel distal enhancer of human adiponectin gene. Promoter constructs with the distal enhancer exhibited high promoter activities in 3T3-L1 mature adipocytes. However, no such activity was observed in other types of cell lines. The distal enhancer is highly conserved, and contains two completely conserved CCAAT boxes. In 3T3-L1 mature adipocytes, deletion or each point mutation of these CCAAT
\end{abstract}

boxes markedly reduced luciferase activity driven by adiponectin promoter. Knockdown of CCAAT/enhancer-binding protein $\alpha$ (CEBPA; also known as $\mathrm{C} / \mathrm{EBP} \alpha$ ) using small interfering RNA diminished adiponectin mRNA expression and luciferase activity driven by adiponectin promoter with the distal enhancer. However, adiponectin promoter with each mutation of two CCAAT boxes in the distal enhancer did not respond to knockdown of CEBPA expression. Furthermore, CEBPA bound to the distal enhancer both in vitro and in vivo. We also identified a proximal promoter region responsible for transcriptional activation by the distal enhancer in human adiponectin gene. Our results indicate that CEBPA plays a pivotal role in the transcription of human adiponectin gene via the distal enhancer and proximal region in its promoter.

Journal of Endocrinology (2009) 200, 107-116

\section{Introduction}

There is sufficient evidence that adipose tissue is not only an energy storage organ, but it produces and secretes a variety of bioactive molecules called adipocytokines, such as tumor necrosis factor $\alpha$, leptin, adiponectin, plasminogen activator inhibitor type-I, resistin, and retinol-binding protein 4 (Hotamisligil \& Spiegelman 1994, Zhang et al. 1994, Maeda et al. 1996, Shimomura et al. 1996, Steppan et al. 2001, Yang et al. 2005). Dysregulated production of adipocytokine is associated with obesity-related metabolic diseases (Matsuzawa et al. 1999). Adiponectin/acrp30/adippoQ is an adipocytokine specifically and abundantly expressed in adipose tissue (Scherer et al. 1995, Hu et al. 1996, Maeda et al. 1996). The normal plasma adiponectin level ranges between 5 and $20 \mu \mathrm{g}$, but the levels are lower in obese subjects, diabetics, and those with ischemic heart disease (Arita et al. 1999). Furthermore, many reports suggest that adiponectin is a key player in the regulation of insulin sensitivity, energy homeostasis, immunological reactions, cardiovascular events and appetite
(Yamauchi et al. 2001, Berg et al. 2002, Maeda et al. 2002, Shibata et al. 2005, Kubota et al. 2007, Takemura et al. 2007).

Precise transcriptional mechanisms of adiponectin gene remain elusive. Previous studies reported that adiponectin is transcriptionally regulated by various transcription factors, such as peroxisome proliferator-activated receptor $\gamma$ (PPARG, also known as PPAR $\gamma$; Iwaki et al. 2003), CCAAT/enhancerbinding protein- $\alpha$ (CEBPA, also known as $\mathrm{C} / \mathrm{EBP} \alpha$; Park et al. 2004, Qiao et al. 2005, Qiao et al. 2006), nuclear factor of activated T cells 4 and activating transcription factor 3 (ATF3; Kim et al. 2006), through cis-regulatory elements in its proximal promoter region, or 1 st intron. However, the $5^{\prime}$ flanking distal region of adiponectin gene has not been fully investigated.

In this study, we analyzed a $10390 \mathrm{bp}$ fragment of the $5^{\prime}$ flanking promoter region of the human adiponectin gene, and found a novel distal enhancer located between $-2667 \mathrm{bp}$ and -2507 bp upstream from its transcription start site. Interestingly, transcriptional activation by this distal enhancer was observed in mature adipocytes but not other types of cell lines. CEBPA bound to this distal enhancer, and regulated the transcriptional 
activity of adiponectin gene. The results also showed that a proximal region in the human adiponectin promoter is necessary for full transcriptional activation by its distal enhancer. These results suggest that CEBPA plays a pivotal role in the transactivation of human adiponectin gene via a communication between the distal and proximal enhancers in its promoter.

\section{Materials and Methods}

\section{Cloning of human adiponectin promoter}

Human adiponectin promoter regions were cloned as reported previously (Segawa et al. 2006). Briefly, human adiponectin promoters $(-10390 /+77,-4075 /+77$, and $-2664 /+77)$ were subcloned into pBSII vector using the Escherichia coli-based bacterial artificial chromosome (BAC) recombination system (Lee et al. 2001). Retrieval cassettes were generated by PCR amplification of $\mathrm{pBSIIKS}+$ vector with primers corresponding to each promoter (these are listed in Supplementary Table 1, see Supplementary data in the online version of the Journal of Endocrinology at http://joe.endocrinology-journals.org/content/vol200/issue1/). The BAC RP23114P23, which contains human adiponectin gene, was obtained from BAC PAC Resources Center (Oakland, CA, USA).

\section{Plasmids}

pGL3 basic and pGL3 promoters were purchased from Promega. a908Luc, a2561Luc, and TK-Luc were previously reported or constructed in our laboratory (Iwaki et al. 2003, Kaneko et al. 2003). Each promoter fragment (-10 390/+77, $-4075 /+77$, and $-2664 /+77)$ was subcloned into pGL3 basic vector (a10390Luc, a4075Luc, and a2664Luc). A serial deleted distal and proximal enhancer region $(-2765 /-2507$, $-2664 /-2507,-2664 /-2539,-2587 /-2507,-563 /+$ $77,-540 /+77,-513 /+77,-449 /+77,-232 /+77$, and $-41 /+77)$ was PCR-amplified using the primers listed in Supplementary Table 1 . The generated proximal fragments were digested with $M l u \mathrm{I}$ and NheI, and inserted into pGL3 basic plasmid (a563Luc, a540Luc, a513Luc, a449Luc, a232Luc, and a41Luc). A serial deleted distal enhancer region was excised with $M l u \mathrm{I}$ and EcoRI, and inserted into a563Luc (a563Luc+ Unit1 (158 bp), a563Luc + Unit2 (81 bp), a563Luc + Unit3 (126 bp), and a563Luc + Unit4 (256 bp)). The proximal fragments were excised with EcoRI and NheI and inserted into a563Luc + Unit1 (a540Luc + Unit1, a513Luc + Unit1, a449Luc + Unit1, a232Luc + Unit1, and a41Luc + Unit1). A2664LucMut1 and a2664LucMut2 were created by PCR mutagenesis using QuickChange II Site-Directed Mutagenesis Kit (Stratagene, La Jolla, CA, USA).

\section{$R N A$ preparations from 3T3-L1 adipocytes}

3T3-L1 cells were maintained in Dulbecco's Modified Eagle's Medium (DMEM) containing 10\% fetal bovine serum (FBS).
For differentiation, cells (3-7 days after confluence) were cultured for 2 days with 10\% FBS-supplemented DMEM containing $5 \mu \mathrm{g} / \mathrm{ml}$ insulin, $0.5 \mathrm{mmol} / 1$ 1-methyl-3-isobutyl-xanthine, and $1 \mu \mathrm{mol} / 1$ dexamethasone. The cells were further incubated with DMEM containing 10\% FBS to differentiate into adipocytes. Total RNA was extracted from differentiated 3T3-L1 adipocytes (on day 8 after induction of differentiation) using RNA-STAT-60 (Tel-Test, Friendswood, TX, USA) using the protocol supplied by the manufacturer.

\section{Quantitative real-time PCR}

The cDNA was synthesized using the ThermoScript RT-PCR system (Invitrogen). Real-time PCR was performed on LightCycler system (FastStart DNA Master SYBR Green I, Roche) according to the protocol provided by the manufacturer. Sequences of primers used for real-time PCR are described in Supplementary Table 1.

\section{$R N A$ interference studies}

3T3-L1 mature adipocytes on day 8 after the induction of differentiation were transfected with $20 \mathrm{nM}$ control and CEBPA small interfering RNA (siRNA) oligonucleotides using DeliverX transfection reagent (Veritas, Tokyo, Japan) following the protocol recommended by the manufacturer. The siRNA oligonucleotides were purchased from Qiagen. The target sequences of CEBPA and negative control are described in Supplementary Table 1.

\section{Luciferase reporter assay}

On day 4 after the induction of differentiation, the medium of 3T3-L1 cells in six-well plates was changed to OPTI-MEM (Invitrogen), and the cells were transfected with luciferase reporter plasmids using Lipofectoamine 2000 reagent (Invitrogen) according to the protocol provided by the manufacturer. Transfection was performed using $1 \mu \mathrm{g}$ pCMX- $\beta$-gal (internal standard) and $2 \mu \mathrm{g}$ reporter plasmids, with or without $20 \mu \mathrm{l}$ of $5 \mu \mathrm{M}$ siRNA solution. Four hours later, the medium was changed to DMEM containing 10\% FBS. After 40-h incubation, luciferase reporter assays were performed using Luciferase Assay system (Promega). Luciferase reporter assay with HEK293 cells was performed as previously described (Iwaki et al. 2003). Transfection efficiencies in different cell types were determined by transfection of green fluorescent protein (GFP)-expressing vector and counting the number of GFP-positive cells. Luciferase values were normalized by an internal $\beta$-galactosidase control and expressed as relative luciferase activity.

\section{Electrophoretic mobility shift assay}

Electrophoretic gel shift assay (EMSA) was performed as described previously (Iwaki et al. 2003) using the double- 
strand nucleotides described in Supplementary Table 1, and anti-CEBPA (\#sc-61, Santa Cruz Biotechnology, Santa Cruz, CA, USA) or non-immune rabbit IgG (\#PP64B, Upstate Biotechnology, Lake Placid, NY, USA). CEBPA protein was synthesized from pcDNA3-HA-CEBPA expression plasmids by the $\mathrm{T}_{\mathrm{N}} \mathrm{T}$ T7 Quick Coupled Transcription/Translation Systems (Promega). The pcDNA3-HA-CEBPA expression plasmid (Kovacs et al. 2003) was a kind gift from Dr Cardinaux. Nuclear extracts were prepared from 3T3-L1 mature adipocytes on days 8-10 after the induction of differentiation using Nuclear Extract Kits (Active Motif, Tokyo, Japan).

\section{Chromatin immunoprecipitation (ChIP) assay}

ChIP assay of 3T3-L1 mature adipocytes on days 8-10 after the induction of differentiation was performed as described previously (Lee et al. 2006) with anti-CEBPA antibody (sc-61), or non-immune IgG (rabbit IgG: Upstate Biotechnology), and the primers described in Supplementary Table 1.

\section{Statistical analysis}

All data were expressed as mean \pm s.D. Differences between groups were examined for statistical significance using the Student's $t$-test. A $P$ value less than $0 \cdot 05$ denoted the presence of a statistically significant difference.

\section{Results}

Human adiponectin promoter exhibits high transcriptional activity in 3T3-L1 mature adipocytes

First, we analyzed the $5^{\prime}$ flanking distal region of human adiponectin gene with UCSC Genome Browser (http:// genome.ucsc.edu). An uncharacterized gene next to adiponectin, AK094454, is located between $31794 \mathrm{bp}$ and $17153 \mathrm{bp}$ upstream from transcription start site of adiponectin gene. Analysis of this $17153 \mathrm{bp}$ fragment revealed the presence of evolutionally conserved regions within $10000 \mathrm{bp}$ upstream from the transcription start site of adiponectin gene (data not shown). Therefore, we cloned a $10390 \mathrm{bp}$ fragment of the $5^{\prime}$ flanking region of human adiponectin gene. Next, to analyze this fragment, luciferase plasmids driven by a serial deletion of adiponectin promoter were constructed, and transfected into 3T3-L1 mature adipocytes. As we have reported previously (Iwaki et al. 2003), a908Luc showed higher luciferase activity than control vectors (pGL3 basic vector containing no promoter and pGL3 promoter vector containing SV40 promoter). Surprisingly, constructs containing long fragments of adiponectin promoter, such as a10 390Luc, a4075Luc, and a2664Luc showed higher luciferase activities than a908Luc (Fig. 1A). Next, to investigate the adiponectin promoter activity in other cell lines, luciferase activities driven by control vectors and reporter constructs with human adiponectin promoters were measured in HEK293 cells. Luciferase activity driven by human adiponectin promoter was apparently lower than that driven by pGL3 promoter vector containing SV40 promoter, and similar to that driven by pGL3 basic vector containing no promoter (Fig. 1B). In other cell lines, such as 3T3-L1 preadipocytes, RAW 264.7 (macrophages), HepG2 (hepatoma) and N1E 115 (neuroblastoma), human adiponectin promoters showed very low promoter activity (Supplemental Figure 1, see Supplementary data in the online version of the Journal of Endocrinology at http://joe. endocrinology-journals.org/content/vol200/issue1/). Collectively, these data suggest that important elements for adipocyte-specific high expression exist between $-2664 \mathrm{bp}$ and $-908 \mathrm{bp}$ upstream from the transcription start site of human adiponectin gene.

\section{Transcriptional activation of adiponectin via the distal enhancer}

Next, to identify the cis-elements responsible for the transactivation in mature adipocytes, the $5^{\prime}$ flanking region of adiponectin gene was analyzed with the UCSC Genome Browser. The UCSC Genome Browser identified five evolutionally conserved regions within the $-2664 \mathrm{bp}$ upstream from the transcription start site of the human adiponectin gene (Fig. 1C). Two conserved elements are located between $-2614 \mathrm{bp}$ and $-2517 \mathrm{bp}$ (Element1 (E1): -2614/-2591 and Element2 (E2): -2524/-2517), and three conserved elements are in the proximal region of human adiponectin promoter (Element3 (E3): $-177 /-158$, Element4 (E4): - 105/ - 92, and Element5 (E5): - 55/-43). To investigate the impact of these two distal conserved elements on the transactivation of human adiponectin promoter, luciferase activities driven by truncated constructs of human adiponectin promoter were measured in 3T3-L1 mature adipocytes. a2664Luc, including E1 and E2, exhibited high luciferase activity; however, the reporter construct deleted E1 (a2561Luc) showed apparently low luciferase activity similar to that driven by the proximal promoter construct with both deleted E1 and E2 (a563; Fig. 1D). This deletion suggested the significance of E1 for the transcriptional activation of human adiponectin promoter in 3T3-L1 mature adipocytes. To define the distal enhancer of the human adiponectin gene, reporter constructs of various truncated distal enhancers with proximal promoter were transfected into 3T3-L1 mature adipocytes. Although connection with the distal enhancer, including both E1 and E2 (a563+Unit1), potently increased the transcriptional activity of a563Luc, slight enhancement was observed by the distal enhancer deleted E1 or E2 (a563 + Unit2 or a563+ Unit3). Taken together, these results suggest that the distal enhancer of human adiponectin gene is composed of highly conserved E1 and E2, and that both are essential for transactivation in mature adipocytes. 
A 3T3-L1 mature adipocyte
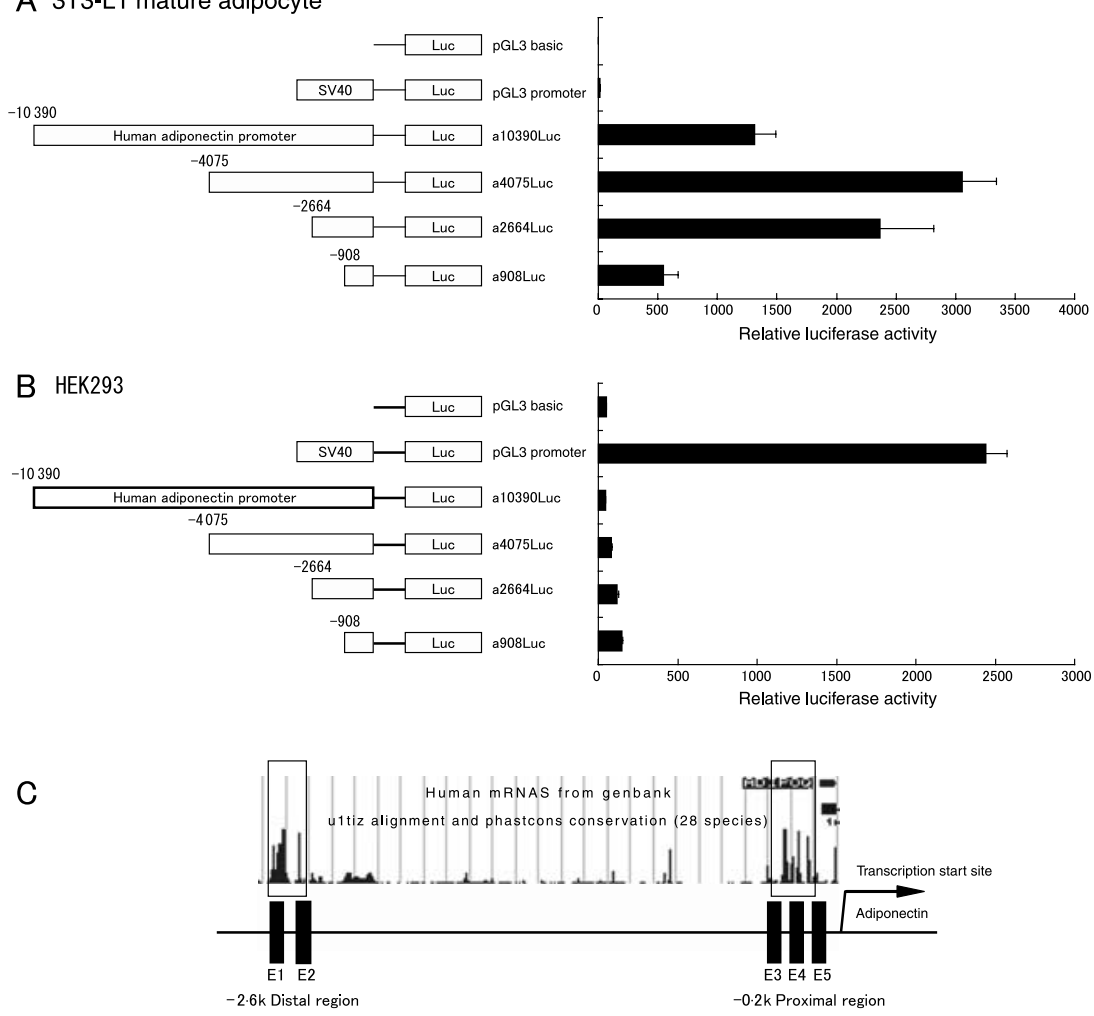

D 3T3-L1 mature adipocyte

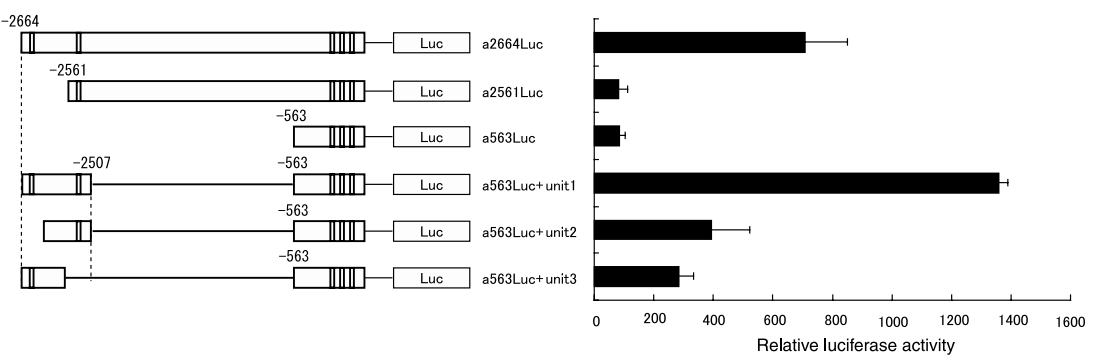

Figure 1 Impact of human adiponectin promoter activity in adipocytes. (A, B, and D) A series of fragments of the $5^{\prime}$-flanking region of the human adiponectin gene were subcloned upstream of the luciferase reporter gene as described in Materials and Methods. Each promoter/reporter construct was transfected into 3T3-L1 mature adipocytes or HEK293 cells, and $48 \mathrm{~h}$ later, luciferase activity was measured and normalized by $\beta$-galactosidase activity. Normalized luciferase activity is expressed as mean \pm s.D. $(n=3)$. (C) The UCSC Genome Browser (http://genome.ucsc.edu) analysis. The histogram shows interspecies homology. Histograms in open squares indicate sites highly conserved between mammals. The arrow indicates the direction of transcription.

\section{CEBPA functionally regulates adiponectin promoter activity} through two conserved CCAAT boxes

As described above, the distal enhancer is composed of conserved E1 and E2 (Fig. 2A), and both are necessary for its transcriptional activation. To identify the transcription factor that binds to E1 and E2, the TRANSFAC database program (http://motif.genome.jp/) was employed. As shown in Fig. 2B, both E1 and E2 were predicted as a CEBPA-binding sites (CCAAT box) with over $85 \%$ identity to its consensus sequence. Furthermore, these putative CCAAT boxes were completely conserved among human, rhesus, mouse, dog, and horse (Fig. 2B). To investigate the importance of these CCAAT boxes in E1 and E2 (C1 and C2 respectively) in the region between $-2507 \mathrm{bp}$ and $-2664 \mathrm{bp}$, mutations that disrupt CEBPA-binding of $\mathrm{C} 1$ and $\mathrm{C} 2$ were introduced into a2664Luc (Fig. 2C). a2664Luc showed higher luciferase activity than a2561Luc, and a mutation on either single C1 or 
A

$-2664$

B

\begin{tabular}{|c|c|c|c|}
\hline Element 1 & CCAAT box1 & Element 2 & CCAAT box2 \\
\hline Human & TCGCAAAATGCTAGGCTITCACAA & Human & TTGTGCAA \\
\hline Rhesus & TCACAAAATGCTAGGCT|TTC & Rhesus & TTGTGCAA \\
\hline Mouse & TCACCAAATGATAGGCगTTC & Mouse & TTGTGCAA \\
\hline Dog & TCA--AAATGACAGGCTTT & Dog & TTGTGCAA \\
\hline \multirow[t]{2}{*}{ Horse } & TCA--AAATGACAGGATTTCA & orse & TTGTGCAA \\
\hline & 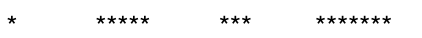 & & \\
\hline
\end{tabular}

C 3T3-L1 mature adipocyte

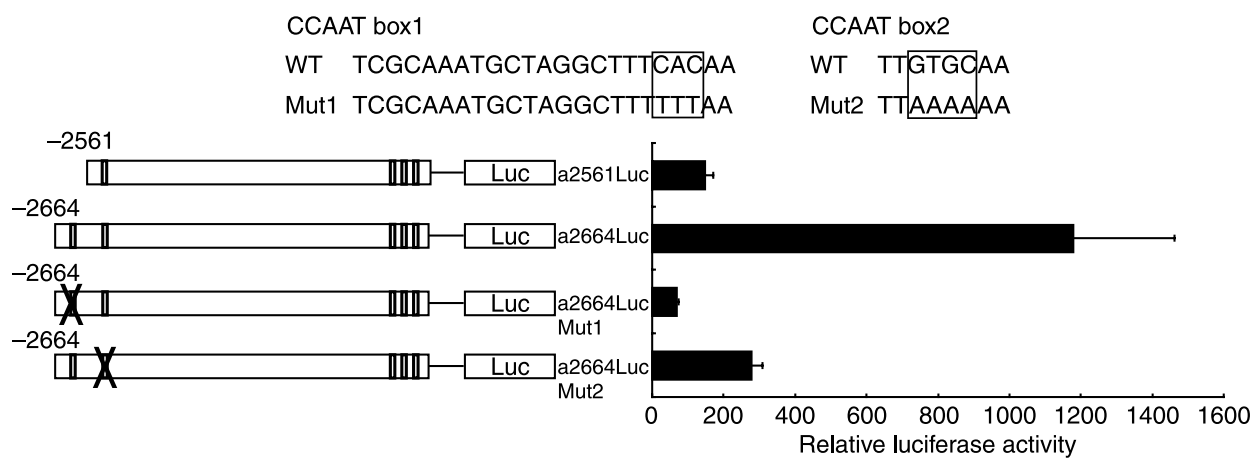

D 3T3-L1 mature adipocyte

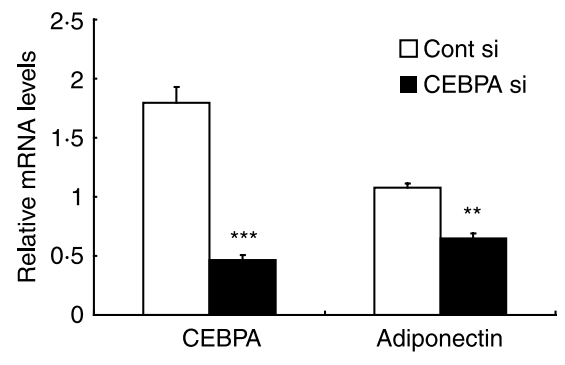

E 3T3-L1 mature adipocyte

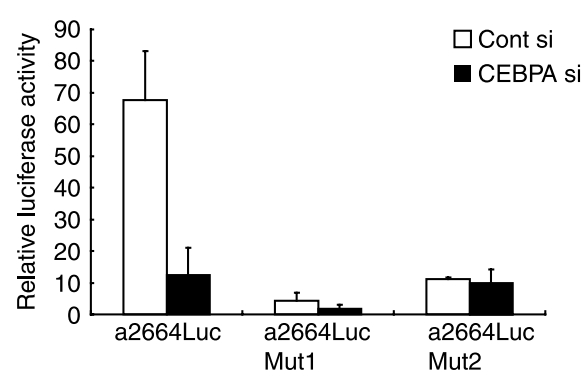

Figure 2 Functional transactivation by CEBPA through two CCAAT boxes in the distal enhancer of human adiponectin promoter. (A) Sequences of $-2664 /-2507$ region of the human adiponectin promoter. Boxed sequences indicate conserved elements demonstrated by UCSC Genome Browser analysis. Arrows indicate the direction of putative CCAAT boxes. (B) Sequences of distal conserved elements in adiponectin gene. The sequences of mammals were obtained from UCSC Genome Browser. Putative CCAAT boxes are enclosed in boxes. Completely conserved nucleotides are represented with asterisks. (C) The indicated reporter constructs with or without mutations in CCAAT box were transfected in 3T3-L1 mature adipocytes. Normalized luciferase activity is expressed as mean \pm s.D. $(n=3)$. Mutated sequences are enclosed in boxes. (D) 3T3-L1 cells on day 8 were transfected with the indicated siRNAs. The mRNA expression levels of CEBPA and adiponectin were measured by quantitative RT-PCR. Values are normalized to the level of 36B4 mRNA and expressed as mean \pm s.D. $(n=3)$. ${ }^{* *} P<0 \cdot 01,{ }^{* * *} P<0 \cdot 001$. (E) WT and mutated reporter constructs were transfected with the indicated siRNAs into 3T3-L1 adipocytes. Normalized luciferase activity is expressed as mean \pm s.D. $(n=3-6)$. 
C2 attenuated the transcriptional activity driven by a2664Luc in 3T3-L1 mature adipocytes. These results indicate that both $\mathrm{C} 1$ and $\mathrm{C} 2$ are responsible for full-transcriptional activation by a2664Luc in 3T3-L1 mature adipocytes. Next, to ask whether the loss of transcriptional activity by mutations of $\mathrm{C} 1$ and $\mathrm{C} 2$ is a CCAAT box-specific effect, other mutant constructs with a random mutations around $\mathrm{C} 1$ or $\mathrm{C} 2$ were transfected into 3T3-L1 mature adipocytes (a2664Luc with a mutation located immediately upstream of C1 and a2664Luc with a mutation located immediately downstream of C2). However, these mutations did not affect the high transcriptional activities driven by a2664Luc (data not shown). Collectively, these data suggest that $\mathrm{C} 1$ and $\mathrm{C} 2$ are important sites in the distal enhancer of human adiponectin gene.

Next, to investigate whether CEBPA functionally regulates adiponectin transcriptional activity through $\mathrm{C} 1$ and $\mathrm{C} 2$, the endogenous expression of CEBPA was suppressed using siRNA in 3T3-L1 mature adipocytes. As reported previously (Qiao et al. 2005), adiponectin mRNA levels in 3T3-L1 mature adipocytes were significantly reduced following suppression of endogenous expression of CEBPA (Fig. 2D). Luciferase activity of a2664Luc was strongly reduced by CEBPA knockdown; however, those of a2664LucMut1 and a2664LucMut2 did not change significantly (Fig. 2E). Taken together, these data suggest that CEBPA regulates human adiponectin transcription through $\mathrm{C} 1$ and $\mathrm{C} 2$ in its conserved distal enhancer.

\section{CEBPA directly binds to CCAAT box in the distal enhancer of adiponectin gene}

To assess the direct binding of CEBPA to C1 and C2 in the human adiponectin gene, EMSA experiments using radiolabeled oligonucleotides including C1 or C2 were employed. Both C1 and C2 bound to in vitro-translated CEBPA, and the mobility of the labeled probes was shifted (Fig. 3A, lanes 2 and 8 ). The signal was attenuated by competition with excess homologous nucleotides (Fig. 3A, lanes 3 and 8), but not with a mutated derivative of these CCAAT boxes, which contains point mutations as described in Supplementary Table 1 (Fig. 3A, lanes 4 and 10). Although oligonucleotides containing consensus CCAAT box of mouse leptin gene were also an effective competitor (Fig. 3A, lanes 5 and 11); no competition was seen by non-functional CCAAT box mutant oligonucleotides reported previously (Hwang et al. 1996; Fig. 3A, lanes 6 and 12).

Next, to investigate whether endogenous nuclear complexes containing CEBPA bind to C1 and C2, we performed EMSA studies with nuclear extracts from 3T3-L1 mature adipocytes. As shown in Fig. 3B, formation of shifted bands was observed with radiolabeled oligonucleotides including C1 or C2 (lanes 1 and 8), which was abrogated by 50 -fold molar excess of unlabeled probes (lanes 2 and 9) or a consensus CCAAT box of mouse leptin promoter (lanes 4 and 11), but not by that of unlabeled mutant CCAAT box probes (lanes 3 and 10) and mutant CCAAT box probes from
A

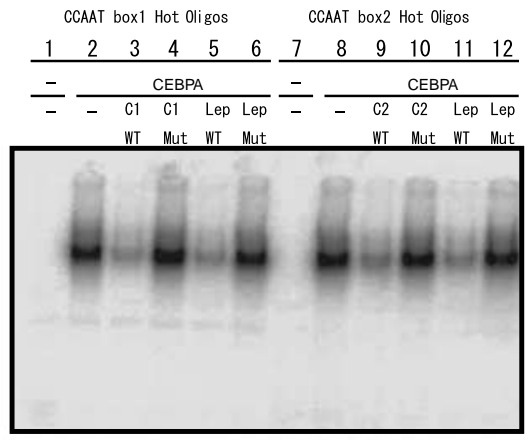

B

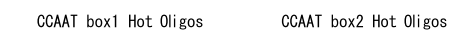

\begin{tabular}{llllllllllllll}
1 & 2 & 3 & 4 & 5 & 6 & 7 \\
\hline
\end{tabular} $\begin{array}{llllll}\text { 3T3-L1 } & \text { Nuc lear Extracts } & \frac{8}{3 T 3-\text { L1 }} \text { Nuc lear Extracts } \\ -\begin{array}{llll}\text { C1 } & \text { C1 Lep Lep Rab CEBPA - C2 } & \text { C2 Lep Lep Rab CEBPA } \\ \text { WT } & \text { Mut WT Mut IgG a Ab } & \text { WT } & \text { Mut WT Mut IgG a Ab }\end{array}\end{array}$

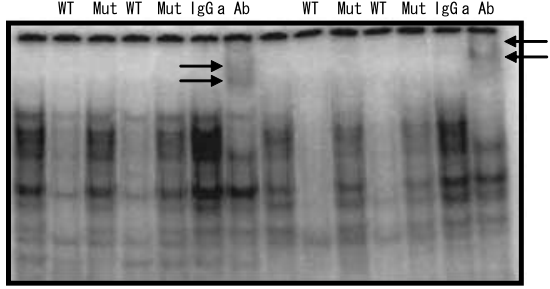

C

\begin{tabular}{|l|l|}
\hline 3T3-L1 preadipocyte & 3T3-L1 mature adipocyte \\
\hline IgG $\begin{array}{l}\text { CEBPA } \\
\text { Ab Input }\end{array}$ & IgG \\
\hline
\end{tabular}

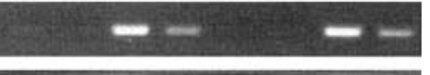

Negative control region

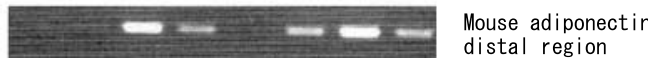

Figure 3 Specific binding of CEBPA to CCAAT boxes in adiponectin distal enhancer. (A) EMSA was performed with $\left[{ }^{32} \mathrm{P}\right]$-labeled oligonucleotides containing the CCAAT box in the distal enhancer region of human adiponectin gene. The indicated labeled probes were incubated with in vitro synthesized CEBPA. Competitive assays were performed using unlabeled oligonucleotides described in results as competitors by 50 -fold molar excess. (B) EMSA was performed with the $\left[{ }^{32} \mathrm{P}\right]$-labeled oligonucleotides mentioned above. The indicated probes were incubated with nuclear extracts from 3T3-L1 mature adipocytes on days $8-10$. Competition assays were performed by adding 50-fold molar excess of unlabeled probes described in results. Non-immune rabbit IgG (Rab IgG) or anti-CEBPA antibody (CEBPA Ab) was added to nuclear extracts from 3T3-L1 adipocytes. Arrows indicate shifted bands of the labeled oligonucleotides bound to CEBPA/antibody complex. (C) In ChIP, chromatin from pre- and mature 3T3-L1 adipocytes on days 8-10 was used. DNA was incubated with CEBPA antibody or non-immune $\operatorname{lgG}$ (IgG), and the recovered DNA was amplified using oligonucleotides flanking these CCAAT boxes or the negative control region. Aliquots of chromatin were analyzed before immunoprecipitation (input).

mouse leptin promoter (lanes 5 and 12). In addition, the specific bands were immunodepleted and supershifted using anti- CEBPA antibody (lanes 7 and 14), but not using nonimmune rabbit IgG (lanes 6 and 13). Finally, in vivo binding of CEBPA to these CCAAT boxes was further verified by ChIP assay. 
Although 3T3-L1 is a cell line derived from mouse, the distal enhancer of the adiponectin gene is highly conserved in human and mouse (Figs 1C and 2B). Fragmented chromatin from formaldehyde cross-linked differentiated 3T3-L1 was subjected to immunoprecipitation with CEBPA antibody or with rabbit IgG as a control. To validate the data, we also performed ChIP with 3T3-L1 preadipocytes, in which CEBPA expression is extremely low (Ramji \& Foka 2002). In 3T3-L1 mature adipocytes, endogenous CEBPA bound to the distal enhancer region of the mouse adiponectin gene, while in 3T3-L1 preadipocytes, CEBPA antibody barely immunoprecipitated the adiponectin distal enhancer (Fig. 3C). In contrast, CEBPA did not immunoprecipitate the sequences from the negative control region in both pre and 3T3-L1 mature adipocytes (Fig. 3C). Taken together, these data strongly suggest that CEBPA binds to the conserved distal enhancer of adiponectin gene in vivo.

\section{Proximal regions of human adiponectin are required for transcriptional activation by the distal enhancer}

During analysis of the distal enhancer of the human adiponectin gene, we found that it could activate its proximal promoter but not a minimal promoter as the herpes simplex virus thymidine kinase promoter (TK-Luc; Fig. 4A). These data led us to hypothesize that the distal enhancer could activate proximal promoter via functional communication in mature adipocytes. To define the communication between proximal and distal enhancers of human adiponectin gene, we performed luciferase assay with various sets of promoterenhancer constructs in 3T3-L1 mature adipocytes. Luciferase activities driven by serial $5^{\prime}$ flanking deletion constructs of proximal adiponectin promoter with or without its distal enhancer were evaluated. As shown in Fig. 4B, the $563 \mathrm{bp}$ and $540 \mathrm{bp}$ proximal promoter constructs (a563Luc and a540Luc) clearly responded to the addition of the distal enhancer (17-fold and 17-fold respectively). However, constructs lacking the sequences between $540 \mathrm{bp}$ and 513 bp apparently reduced the transcriptional activation by the distal enhancer (a513Luc, a449Luc, a232Luc, and a91Luc; 6-folds, 6-folds, 7-folds, and 4-folds respectively; Fig. 4B). These data suggest the importance of sequences between $540 \mathrm{bp}$ and $513 \mathrm{bp}$ of human adiponectin gene for fulltranscriptional activation by its distal enhancer.

\section{Discussion}

Adiponectin is exclusively expressed in adipocytes of both rodents and human (Scherer et al. 1995, Hu et al. 1996, Maeda et al. 1996); however, little is known about the mechanisms underlying this adipocyte-specific expression. In the present study, we identified two highly conserved CCAAT boxes in the $5^{\prime}$ flanking distal region of human adiponectin gene. CEBPA bound to these CCAAT boxes and enhanced the transcriptional activities of human adiponectin promoter via a unique communication with its proximal promoter region. This proximal region located between $540 \mathrm{bp}$ and $513 \mathrm{bp}$ of human adiponectin gene, designated as 'the distal enhancerresponse element', seems to play an important role.

The CEBP family is considered a key class of transcription factors in adipogenesis. Briefly, CEBPB and CEBPD are induced during the mitotic clonal expansion phase of preadipocytes in response to differentiation reagents. Then, the preadipocytes exit the cell cycle and start to express CEBPA. CEBPA has been implicated as a regulator of the integrity of adipocytes via induction of various adipose-marker genes (Mandrup \& Lane 1997, Ramji \& Foka 2002), including fatty acid-binding protein 4 (aP2), leptin, resistin, $\beta 3$-adrenergeic receptor, and PPARG2 (Hwang et al. 1996, Mason et al. 1998, Elberg et al. 2000, Dixon et al. 2001, Hartman et al. 2002, Tuncman et al. 2006). Using siRNA of CEBPA, the present study highlighted the involvement of CEBPA in adiponectin expression. This result is in agreement with the findings of previous studies, which demonstrated that overexpression of CEBPA upregulates adiponectin mRNA levels in both human and mouse adipocytes, and that the knockdown of CEBPA expression reduces adiponectin mRNA levels both in human and mouse adipocytes (Qiao et al. 2005).

Although the significance of CEBPA on adiponectin gene expression has been reported, the regulatory mechanism of adiponectin gene expression by CEBPA has been controversial. Park et al. (2004) demonstrated that CEBPA could bind to both mouse and human adiponectin proximal promoters and increase the activities of these promoters. On the other hand, others reported that ectopic expression of CEBPA could not upregulate luciferase activities driven by the proximal promoters of mouse and human adiponectin gene respectively (Seo et al. 2004, Qiao et al. 2005). Qiao et al. (2005) reported that an intronic enhancer of human adiponectin gene is important for this regulation, although no homology was seen in the first intron of human and mouse adiponectin gene. The present study demonstrated that other CEBPA-binding sites in the distal $5^{\prime}$ flanking region of the adiponectin gene are significant, and completely conserved among human, mouse, rhesus, dog, and horse. Furthermore, siRNA-mediated reporter assay showed that CEBPA functionally regulates human adiponectin transcriptional activity through CCAAT boxes in its distal enhancer. Collectively, CEBPA should be involved in adipose tissue expression of adiponectin mainly through this distal enhancer.

As demonstrated in Fig. 1A and B, the human adiponectin promoter, including its distal enhancer, exhibited high transcriptional activities only in mature adipocytes among various cell lines investigated in this study (Supplemental Figure 1). To investigate the mechanism underlying this cell line-specific induction, adiponectin promoter activity was measured in HEK293 cells overexpressing CEBPA. However, the transcriptional activity driven by any length of human adiponectin promoter did not significantly respond to ectopic 
A 3T3-L1 mature adipocyte

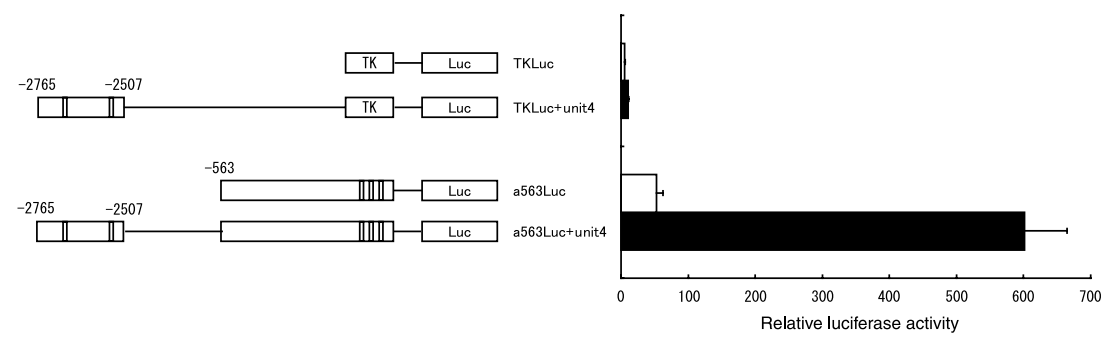

B 3T3-L1 mature adipocyte

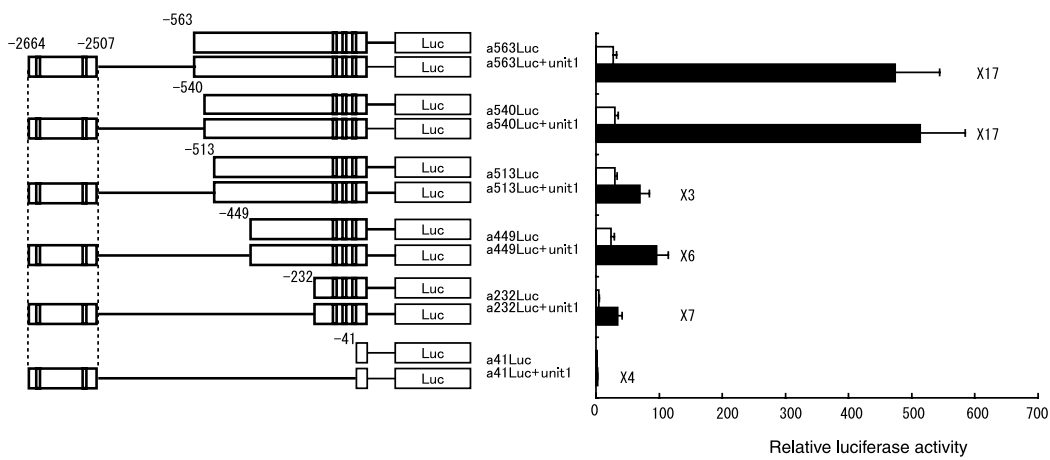

C

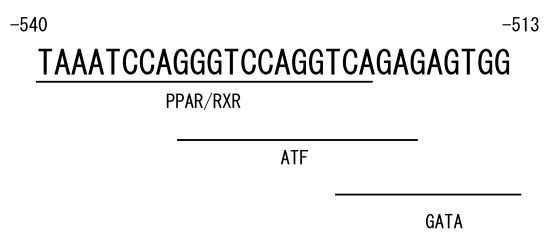

Figure 4 Identification of the distal enhancer-response element in the proximal promoter of human adiponectin gene. (A) The indicated reporter constructs were transfected in 3T3-L1 mature adipocytes, and $48 \mathrm{~h}$ later, luciferase activity was measured and normalized by $\beta$-galactosidase activity. Normalized luciferase activity is expressed as mean \pm s.D. $(n=3)$. (B) The indicated reporter constructs were transfected in 3T3-L1 mature adipocytes and $48 \mathrm{~h}$ later, luciferase activity was measured and normalized by $\beta$-galactosidase activity. Normalized luciferase activity is expressed as mean \pm s.D. $(n=3)$. (C) Sequences of the distal enhancer-response element in the proximal promoter of human adiponectin gene. The underlined sequences represent putative binding sites of the indicated transcription factors.

CEBPA expression in HEK293 cells (data not shown). In fact, CEBPA is expressed at high levels in the liver and lung, in which no adiponectin expression is detected. These findings indicate that CEBPA per se is unable to increase adiponectin transcription, and not enough to attain adipocyte-specific expression of adiponectin. Exactly how the specificity of adiponectin gene is determined remains an unanswered question. Previously, we and others reported that PPARG regulates adiponectin mRNA level and promoter activity in mature adipocytes (Maeda et al. 2001, Iwaki et al. 2003, Qiao et al. 2005). PPARG is strongly expressed in mature adipocytes, and plays a pivotal role on differentiation of adipocytes (Rosen \& Spiegelman 2001). In this regard, synergistic activation of adiponectin gene by both CEBPA and PPAR G may, at least partly, explain its adipocyte-specific expression.

Interestingly, the distal enhancer in human adiponectin gene enhances the transcriptional activity driven by its proximal promoter, but not by minimal promoter like the TK promoter in 3T3-L1 mature adipocytes (Fig. 4A). Furthermore, our data indicated the presence of a 'distal enhancer-response element' in the proximal promoter, which should play a role in communication with the distal enhancer (Fig. 4B). A search for putative cis-elements in 'the distal enhancer-response element' with TRANSFAC database program identified putative binding sites of several transcription factors (PPAR/RXRbinding site, ATF-binding site, and GATA-binding site; Fig. 4C). Previously, we reported that PPARG regulates 
adiponectin mRNA levels and promoter activities in mature adipocytes (Iwaki et al. 2003). As mentioned above, PPARG is strongly expressed in mature adipocytes and plays a pivotal role in transactivation of adiponectin gene. In this regard, we focused on the putative PPAR/RXR-binding site among the predicted transcription factors. However, mutations on this site that should have disrupted PPARG/RXA $\alpha$ binding did not attenuate PPARG agonist-mediated transcriptional activity driven by a2664Luc in 3T3-L1 mature adipocytes (data not shown), indicating that this putative PPAR/RXRbinding site is not functional in terms of the transcriptional activation by PPARG/RXR $\alpha$ heterodimer. On the other hand, the functional PPARG response element, which was identified previously by our group (Iwaki et al. 2003), contributed to PPARG/RXR $\alpha$-mediated transcriptional activation of human adiponectin gene. What factors and/or complexes bind to 'the distal enhancer-response element' in human adiponectin gene remain to be elucidated, and that may be a unique target to increase adiponectin as an anti-diabetic and anti-atherogenic adipocytokine.

In summary, the present study demonstrated that CEBPA plays a crucial role in the transcriptional regulation of human adiponectin gene via the distal enhancer region containing two functional CEBPA-binding sites. This distal enhancer region of adiponectin gene is highly conserved among species. We also identified a distal enhancer-response element in its proximal promoter region. Communication between the distal enhancer and distal enhancer-response proximal elements may play an important role in the adipocyte-specific expression of adiponectin gene.

\section{Declaration of interest}

The authors declare that they have no competing financial interests.

\section{Funding}

This work was supported by grants from the Ministry of Health, Labor and Welfare, Japan, grants from the Ministry of Education, Culture, Sports, Science and Technology, and grants from Takeda Science Foundation.

\section{Acknowledgements}

We thank Dr Makoto Makishima and Dr D J Mangelsdorf for generously providing the expression plasmids pCMX- $\beta$-gal and TK-Luc. The authors also thank Sachiyo Tanaka for her excellent technical help. We are also indebted to all members of Shimomura's laboratory for their helpful discussion.

\section{References}

Arita Y, Kihara S, Ouchi N, Takahashi M, Maeda K, Miyagawa J, Hotta K, Shimomura I, Nakamura T, Miyaoka K et al. 1999 Paradoxical decrease of an adipose-specific protein, adiponectin, in obesity. Biochemical and Biophysical Research Communications 257 79-83.
Berg AH, Combs TP \& Scherer PE 2002 ACRP30/adiponectin: an adipokine regulating glucose and lipid metabolism. Trends in Endocrinology and Metabolism 13 84-89.

Dixon TM, Daniel KW, Farmer SR \& Collins S 2001 CCAAT/enhancerbinding protein $\alpha$ is required for transcription of the $\beta 3$-adrenergic receptor gene during adipogenesis. Journal of Biological Chemistry 276 722-728.

Elberg G, Gimble JM \& Tsai SY 2000 Modulation of the murine peroxisome proliferator-activated receptor gamma 2 promoter activity by CCAAT/enhancer-binding proteins. Journal of Biological Chemistry 275 27815-27822.

Hartman HB, Hu X, Tyler KX, Dalal CK \& Lazar MA 2002 Mechanisms regulating adipocyte expression of resistin. Journal of Biological Chemistry 277 19754-19761.

Hotamisligil GS \& Spiegelman BM 1994 Tumor necrosis factor alpha: a key component of the obesity-diabetes link. Diabetes 43 1271-1278.

Hu E, Liang P \& Spiegelman BM 1996 AdipoQ is a novel adipose-specific gene dysregulated in obesity. Journal of Biological Chemistry 271 10697-10703.

Hwang CS, Mandrup S, MacDougald OA, Geiman DE \& Lane MD 1996 Transcriptional activation of the mouse obese (ob) gene by CCAAT/ enhancer binding protein alpha. PNAS 93 873-877.

Iwaki M, Matsuda M, Maeda N, Funahashi T, Matsuzawa Y, Makishima M \& Shimomura I 2003 Induction of adiponectin, a fat-derived antidiabetic and antiatherogenic factor, by nuclear receptors. Diabetes 52 1655-1663.

Kaneko E, Matsuda M, Yamada Y, Tachibana Y, Shimomura I \& Makishima M 2003 Induction of intestinal ATP-binding cassette transporters by a phytosterol-derived liver X receptor agonist. Journal of Biological Chemistry 278 36091-36098.

Kim HB, Kong M, Kim TM, Suh YH, Kim WH, Lim JH, Song JH \& Jung MH 2006 NFATc4 and ATF3 negatively regulate adiponectin gene expression in 3T3-L1 adipocytes. Diabetes 55 1342-1352.

Kovacs KA, Steinmann M, Magistretti PJ, Halfon O \& Cardinaux JR 2003 CCAAT/enhancer-binding protein family members recruit the coactivator CREB-binding protein and trigger its phosphorylation. Journal of Biological Chemistry 278 36959-36965.

Kubota N, Yano W, Kubota T, Yamauchi T, Itoh S, Kumagai H, Kozono H, Takamoto I, Okamoto S, Shiuchi T et al. 2007 Adiponectin stimulates AMP-activated protein kinase in the hypothalamus and increases food intake. Cell Metabolism 6 55-68.

Lee EC, Yu D, Martinez de Velasco J, Tessarollo L, Swing DA, Court DL, Jenkins NA \& Copeland NG 2001 A highly efficient Escherichia coli-based chromosome engineering system adapted for recombinogenic targeting and subcloning of BAC DNA. Genomics 73 56-65.

Lee TI, Johnstone SE \& Young RA 2006 Chromatin immunoprecipitation and microarray-based analysis of protein location. Nature Protocols 1 729-748.

Maeda K, Okubo K, Shimomura I, Funahashi T, Matsuzawa Y \& Matsubara K $1996 \mathrm{cDNA}$ cloning and expression of a novel adipose specific collagen-like factor, apM1 (adipose most abundant gene transcript 1). Biochemical and Biophysical Research Communications 221 286-289.

Maeda N, Takahashi M, Funahashi T, Kihara S, Nishizawa H, Kishida K, Nagaretani H, Matsuda M, Komuro R, Ouchi N et al. 2001 PPARgamma ligands increase expression and plasma concentrations of adiponectin, an adipose-derived protein. Diabetes 50 2094-2099.

Maeda N, Shimomura I, Kishida K, Nishizawa H, Matsuda M, Nagaretani H, Furuyama N, Kondo H, Takahashi M, Arita Y et al. 2002 Diet-induced insulin resistance in mice lacking adiponectin/ACRP30. Nature Medicine 8 731-737.

Mandrup S \& Lane MD 1997 Regulating adipogenesis. Journal of Biological Chemistry 272 5367-5370.

Mason MM, He Y, Chen H, Quon MJ \& Reitman M 1998 Regulation of leptin promoter function by $\mathrm{Sp} 1, \mathrm{C} / \mathrm{EBP}$, and a novel factor. Endocrinology 139 1013-1022.

Matsuzawa Y, Funahashi T \& Nakamura T 1999 Molecular mechanism of metabolic syndrome $\mathrm{X}$ : contribution of adipocytokines adipocytederived bioactive substances. Annals of the New York Academy of Sciences 892 146-154.

Park SK, Oh SY, Lee MY, Yoon S, Kim KS \& Kim JW 2004 CCAAT/enhancer binding protein and nuclear factor-Y regulate adiponectin gene expression in adipose tissue. Diabetes 53 2757-2766. 
Qiao L, Maclean PS, Schaack J, Orlicky DJ, Darimont C, Pagliassotti M, Friedman JE \& Shao J 2005 C/EBPalpha regulates human adiponectin gene transcription through an intronic enhancer. Diabetes 54 1744-1754.

Qiao L, Schaack J \& Shao J 2006 Suppression of adiponectin gene expression by histone deacetylase inhibitor valproic acid. Endocrinology 147 865-874.

Ramji DP \& Foka P 2002 CCAAT/enhancer-binding proteins: structure, function and regulation. Biochemical Journal 365 561-575.

Rosen ED \& Spiegelman BM 2001 PPARgamma: a nuclear regulator of metabolism, differentiation, and cell growth. Journal of Biological Chemistry 276 37731-37734.

Scherer PE, Williams S, Fogliano M, Baldini G \& Lodish HF 1995 A novel serum protein similar to C1q, produced exclusively in adipocytes. Journal of Biological Chemistry 270 26746-26749.

Segawa K, Fukuhara A, Hosogai N, Morita K, Okuno Y, Tanaka M, Nakagawa Y, Kihara S, Funahashi T, Komuro R et al. 2006 Visfatin in adipocytes is upregulated by hypoxia through HIF1alpha-dependent mechanism. Biochemical and Biophysical Research Communications 349 875-882.

Seo JB, Moon HM, Noh MJ, Lee YS, Jeong HW, Yoo EJ, Kim WS, Park J, Youn BS, Kim JW et al. 2004 Adipocyte determination- and differentiation-dependent factor $1 /$ sterol regulatory element-binding protein $1 \mathrm{c}$ regulates mouse adiponectin expression. Journal of Biological Chemistry 279 22108-22117.

Shibata R, Sato K, Pimentel DR, Takemura Y, Kihara S, Ohashi K, Funahashi T, Ouchi N \& Walsh K 2005 Adiponectin protects against myocardial ischemiareperfusion injury through AMPK- and COX-2-dependent mechanisms. Nature Medicine 11 1096-1103.

Shimomura I, Funahashi T, Takahashi M, Maeda K, Kotani K, Nakamura T, Yamashita S, Miura M, Fukuda Y, Takemura K et al. 1996 Enhanced expression of PAI-1 in visceral fat: possible contributor to vascular disease in obesity. Nature Medicine 2 800-803.
Steppan CM, Bailey ST, Bhat S, Brown EJ, Banerjee RR, Wright CM, Patel HR, Ahima RS \& Lazar MA 2001 The hormone resistin links obesity to diabetes. Nature 409 307-312.

Takemura Y, Ouchi N, Shibata R, Aprahamian T, Kirber MT, Summer RS, Kihara S \& Walsh K 2007 Adiponectin modulates inflammatory reactions via calreticulin receptor-dependent clearance of early apoptotic bodies. Journal of Clinical Investigation 117 375-386.

Tuncman G, Erbay E, Hom X, De Vivo I, Campos H, Rimm EB \& Hotamisligil GS 2006 A genetic variant at the fatty acid-binding protein aP2 locus reduces the risk for hypertriglyceridemia, type 2 diabetes, and cardiovascular disease. PNAS 103 6970-6975.

Yamauchi T, Kamon J, Waki H, Terauchi Y, Kubota N, Hara K, Mori Y, Ide T, Murakami K, Tsuboyama-Kasaoka N et al. 2001 The fat-derived hormone adiponectin reverses insulin resistance associated with both lipoatrophy and obesity. Nature Medicine 7 941-946.

Yang Q, Graham TE, Mody N, Preitner F, Peroni OD, Zabolotny JM, Kotani K, Quadro L \& Kahn BB 2005 Serum retinol binding protein 4 contributes to insulin resistance in obesity and type 2 diabetes. Nature 436 356-362.

Zhang Y, Proenca R, Maffei M, Barone M, Leopold L \& Friedman JM 1994 Positional cloning of the mouse obese gene and its human homologue. Nature 372 425-432.

Received in final form 7 October 2008

Accepted 9 October 2008

Made available online as an Accepted Preprint 16 October 2008 